\title{
Thermodynamic and Conformational Properties of Styrene-Methyl Methacrylate Block Copolymers in Dilute Solution. IV. Behavior of Diblock and Triblock Copolymers in Selective Solvents*
}

\author{
Tadao Kotaka, Takeshi Tanaka, and Hiroshi InAGaki \\ Institute for Chemical Research, Kyoto University, Uji, Kyoto-fu 611, Japan.
}

(Received August 10, 1971)

\begin{abstract}
Solution properties of nearly equimolar AB-diblock and BAB-triblock copolymers, wherein A is polystyrene (PST) and B poly(methyl methacrylate) (PMMA), were examined in various selective solvents. In either type of selective solvents the diblock copolymers usually underwent intermolecular association and formed micelles, in which domains of PST- and PMMA-subchains were presumably segragated (intramolecular phase separation). The stability and size of such micelles depended on their molecular weight and composition as well as on the nature of solvents. The behavior of the triblock copolymers appeared to be more critically influenced by the solubility of the PMMA than that of the PST. In selective solvents having preferential solvency towards PMMA rather than PST, their behavior was more or less similar to that of the diblock copolymers whilst in selective solvents preferential to PST, they were usually unstable and liable to precipitate. In the transition region between the stable solution and precipitation states, the individual triblock copolymer chains often showed conformational anomalies without forming micelles, as shown by the intrinsic viscosity anomaly in $p$-xylene at $30^{\circ} \mathrm{C}$. The possibility of intramolecular association of two PMMA subchains within a single triblock copolymer molecule was suggested as a hypothesis. However the hydrodynamic properties alone cannot provide decisive evidence for this possibility.
\end{abstract}

KEY WORDS Block Copolymer / Solution Behavior / Selective Solvent System / AB-diblock Copolymer / BAB-triblock Copolymer / Intramolecular Phase Separation / Intermolecular Association / Micelle Formation / Intramolecular Association / Conformational Anomaly /

In earlier articles ${ }^{1-4}$ of this series the dilute solution properties of styrene (ST) and methyl methacrylate (MMA) block copolymers were described. The materials studied were mainly a series of triblock copolymers of poly(methyl methacrylate) - polystyrene - poly(methyl methacrylate) type having nearly equimolar composition but differing molecular weights. Upon examining their intrinsic viscosity versus molec-

\footnotetext{
* Part of this work was presented at the 160 th National Meeting of the American Chemical Society, Division of Polymer Chemistry, Chicago, Illinois, September, 1970; and also at the 19 th Polymer Symposium of the Society of Polymer Science, Japan, Kyoto, October, 1970.
}

ular weight relationships in various solvents, a significant anomaly (especially in selective solvents of the type favourable towards polystyrene (PST)) was found ${ }^{3-6}$. This anomaly is likely to have resulted from the intramolecular interaction of the two side PMMA subchains. If such interaction is in fact to be the cause of the anomaly, the behavior of diblock copolymers of PST-PMMA type should have been different from PMMA-PST-PMMA triblock copolymers. In selective solvents PST-PMMA diblock copolymers might undergo intermolecular association $^{7}$ rather than the intramolecular interaction, forming stable micelles even in dilute solution; ${ }^{8}$ and the micelle formation should 
have occurred in either type of selective solvents, i.e., in either of those having solubility preferential towadrs PMMA or PST. This behavior is to be contrasted to that of PMMA-PSTPMMA triblock copolymers: their solubility was found to be more affected by side PMMA subchains rather than by the central PST subchain. ${ }^{5}$ In order to clarify these differences between PST-PMMA diblock and PMMAPST-PMMA triblock copolymers, their dilute solution behavior, especially the intrinsic viscosity and sedimentation equilibrium data, was examined in certain selective solvents. The results are reported herein.

\section{EXPERIMENTAL}

\section{Polymer Samples}

Triblock copolymers of PMMA-PST-PMMA type were prepared by an anionic polymerization technique..$^{9-13}$ The preparation was carried out at $-78^{\circ} \mathrm{C}$ in tetrahydrofuran (THF) medium with an initiator (sodium biphenyl/THF solution). Diblock copolymers of PST-PMMA type were prepared also by an anionic polymerization technique: the procedure was the same as for the triblock copolymers except that $n$-butyllithium/n-hexane solution was used as the initiator. The initiator solution was prepared by following the method reported by Fujimoto, Ozaki, and Nagasawa. ${ }^{13}$

The preparation was carried out through the following steps as described before. ${ }^{2,5}$ First the initiator was allowed to react with ST-monomer in THF medium, yielding 'living' PST. An aliquot of the living PST was recovered as a reference homo-PST sample. A small amount of 1,1-diphenylethylene was added to the rest of the living PST solution to replace terminal styryl carbanions by less reactive diphenylmethyl carbanions. This step was employed, as suggested by Freyss, Rempp, and Benoit, ${ }^{12}$ to suppress grafting of living PST to the ester groups of the MMA units when they were added subsequently. Then MMA monomer was introduced, and the living PST initiated the polymerization of the MMA, yelding a block copolymer sample.

As surmised from the procedure described above, the triblock samples might be con- taminated by homo-PST, PST-PMMA diblock copolymers and, far less likely but still possibly, by homo-PMMA; whereas the diblock samples might be contaminated by homo-PST and, less likely but still possibly, by homo-PMMA. As suggested by Urwin and Stearne, ${ }^{11}$ each crude sample of the triblock type was subjected to extraction with boiling cyclohexane to remove free PST, thoroughly dried, and then subjected to extraction with boiling acetonitrile to remove free PMMA. On the other hand, the boilingpoint extraction posed a problem when applied to diblock samples. As will be shown later, the PST-PMMA samples were often dissolved by forming micelles in either cyclohexane or acetonitrile at an elevated temperature, and were lost into the extractant. Therefore each diblock sample was treated simply by washing with cyclohexane at room temperature and sacrificing part of the sample.

A few homo-PMMA samples were prepared by the same anionic polymerization technique using sodium biphenyl/THF initiator. ${ }^{2}$

\section{Measurements}

Characterization of the polymer samples was carried out according to the authors' laboratory routine which was described earlier. ${ }^{2-6,14,15}$ Monomer composition was determined by combustion analysis. Osmotic pressure measurements were made on a Mechrolab high-speed membrane-osmometer model 502. Light scattering measurements were made on a Shimadzu light-scattering photometer of modified Brice type. ${ }^{14}$ Determination of intrinsic viscosity $[\eta]$ was made on Ubbelohde dilution viscometers. For determining critical miscibility temperature $T_{\mathrm{c}}$ of block copolymer solutions, a solubility test was made according to the procedure which was described earlier. ${ }^{3}$

Sedimentation equilibrium experiments ${ }^{16-20}$ were carried out on a Hitachi analytical ultracentrifuge model UCA-1A equipped with a Rayleigh interference and a schlieren optics. ${ }^{21}$ Alignment and forcusing of the optical systems were preformed according to a procedure that is essentially similar to the one developed by LaBar and Baldwin ${ }^{18}$ for a Spinco model $E$ ultracentrifuge. Yphantis six-channel cells ${ }^{19}$ were employed. For the runs with rotor speeds 
higher than $6,020 \mathrm{rpm}$ a standard rotor RA-60H was used; for the runs below this speed a sixplace heavy rotor with four blind and one Rayleigh interference counterbalances was used to attain better rotational stability.

Interference fringe patterns were analyzed ${ }^{18}$ according to the procedure that had been modified to treat heterogeneous samples which contain polymer species having different molecular weights and compositions. ${ }^{21}$ From each equilibrium pattern, cell-averaged molecular weight $\bar{M}_{1}^{\text {app }}$ was calculated as

$$
\begin{gathered}
\bar{M}_{1}^{\mathrm{app}}=[n(b)-n(a)] / \lambda n^{0} \\
\lambda=\left(1-\bar{v}^{0} \rho_{\mathrm{s}}\right) \omega^{2}\left(b^{2}-a^{2}\right) / 2 R T
\end{gathered}
$$

Here $n(a), n(b)$, and $n^{0}=\nu^{0} c^{0}$ are, respectively, the local equilibrium concentrations at the meniscus and at the bottom of the solution column and the concentration of the original (uniform) solution, all expressed in terms of a refractive-index increment scale; $\nu^{0}$ and $\bar{v}^{0}$ are the specific refractive index increment and the partial specific volume of the original sample, respectively; $c^{0}$ is the original solute concentration in $(\mathrm{g} / \mathrm{m} l)$ scale; $\rho_{\mathrm{S}}$ is the solvent viscosity; $\omega$ is the rotor speed in ( $\operatorname{radian} / \mathrm{sec}) ; a$ and $b$ are the radial distances of the meniscus and the bottom of the solution column, respectively; $R T$ is the gas constant times the absolute temperature; $\lambda$ is the sedimentation parameter as defined by eq 2 .

The cell-averaged molecular weight $\bar{M}_{1}^{\text {app }}$ may be extrapolated to infinite dilution according to the equation

$$
\left(\bar{M}_{1}^{\text {app }}\right)^{-1}=\left(\bar{M}_{1}\right)^{-1}+c^{0} B_{\text {app }}(\lambda)+\cdots
$$

Here $\bar{M}_{1}$ is the apparent molecular weight; ${ }^{17,21,22}$ and $B_{\text {app }}(\lambda)$ is the apparent second virial coefficient which depends on the parameter $\lambda^{21}$ Therefore, for securing a better estimation of $\bar{M}_{1}$, the dual extraporation of $\left(\bar{M}_{1}^{\text {app }}\right)^{-1}$ with respect to $\lambda$ and $c^{0}$ is often necessary. ${ }^{20}$

The quantity $\bar{M}_{1}$ depends on the nature of solvent through the variation of partial specific volumes and specific refractive-index increments of the heterogeneous solute, and hence, reflects the compositional heterogeneity of the copolymer sample. ${ }^{21,22}$ If the sample is compositionally homogeneous, the quantity $\bar{M}_{1}$ becomes identical to the weight-average molecular weight $M_{w}{ }^{17,21}$
On the other hand, if the sample is compositionally heterogeneous, equilibrium experiments must be made in several different solvents to deduce its true $M_{w}$. The situation is entirely similar to the analysis of light-scattering apparent molecular weights. ${ }^{23,24}$

\section{RESULTS*}

\section{Characterization of Polymer Samples}

Characterization data of the polymer samples used in this study are listed in the relevant tables shown below. The block copolymer samples were coded as series $B$, and their precursor homo-PST as series $H$. Each block copolymer and its precursor homo-PST was given the same code number for the sake of easy recognition. Homo-PMMA samples were coded as series $\mathrm{M}$. The determination of $M_{w}$ for the triblock copolymer samples was described in detail in a previous paper. ${ }^{2}$ Values of $M_{w}$ for the diblock copolymer samples were determined by sedimentation equilibrium experiments in various solvents of different refractive indices, (toluene and diethyl malonate) and also by light scattering in diethyl malonate. The result is given in Tables II and III. Each block copolymer sample was found to be reasonably homogeneous in molecular weight, composition and chain architecture: ${ }^{2}$ each sample contained only linear chains of the anticipated block number and was not contaminated by branched chains such as those having PST-subchains grafted to PMMA-subchains.

\section{Results of Solubility Test}

Results of the solubility test on several samples of PST-PMMA diblock and PMMA-PSTPMMA triblock type are summarized in Table I, which lists the values of either the critical miscibility temperature $T_{\mathrm{c}}$ or the ' $\Theta$ ' temperature. $^{25}$ The ' $\Theta$ ' temperature was determined ${ }^{1,6}$ as the temperature at which the osmotic second virial coefficient $A_{2}$ vanishes, or the $T_{\mathrm{c}}$ in the limit of infinitely large molecular weight. $^{25}$ Block copolymer samples often gave rise to visibly turbid solutions as indicated in Table I, especially when they were dissolved in selective solvents. Nevertheless macroscopic phase sepation had not taken place from such visibly

* Numerical data are available upon request. 
Table I. Critical miscibility temperature $T_{\mathrm{c}}$ or $\Theta$-temperature $\left({ }^{\circ} \mathrm{C}\right)$ for PST-PMMA diblock and PMMA-PST-PMMA triblock copolymers in various solvents ${ }^{\mathrm{a}}$

\begin{tabular}{|c|c|c|c|c|c|c|c|c|c|c|c|}
\hline \multirow{4}{*}{$\begin{array}{l}\text { Code } \\
10^{-4} M_{n} \\
\text { ST-mole fract }\end{array}$} & \multirow{2}{*}{\multicolumn{2}{|c|}{$\begin{array}{cc}\text { PMMA } & \text { PST } \\
14 \mathrm{M} & 16 \mathrm{H}\end{array}$}} & \multicolumn{3}{|c|}{ PST-PMMA } & \multicolumn{6}{|c|}{ PMMA-PST-PMMA } \\
\hline & & & $39 \mathrm{~B}$ & 47B & 46B & 20B & 28B & 26B & $15 \mathrm{~B}$ & $16 \mathrm{~B}$ & $11 \mathrm{~B}$ \\
\hline & 7.2 & 20.6 & 5.5 & 11.7 & 34.0 & 16 & 7.0 & 26.0 & 31.7 & 39.0 & 19.3 \\
\hline & 0 & 1.00 & 0.48 & 0.42 & 0.45 & 0.36 & 0.46 & 0.45 & 0.49 & 0.72 & 0.85 \\
\hline Cyclohexane & $\mathrm{N}$ & $\Theta=35$ & 20 & $((100))$ & $\mathbf{N}$ & $\mathbf{N}$ & $\mathrm{N}$ & $\mathrm{N}$ & $\mathbf{N}$ & $\mathrm{N}$ & $\mathbf{N}$ \\
\hline trans-Decalin & $\mathrm{N}$ & $\Theta=18$ & $(\mathbf{S})$ & $((\mathbf{S}))$ & $\mathrm{N}$ & $\mathrm{N}$ & $\mathrm{N}$ & $\mathrm{N}$ & $\mathrm{N}$ & $\mathrm{N}$ & - \\
\hline 1-Chloro- $n$-dedecane & $\mathrm{N}$ & 39 & $\mathrm{~N}$ & $\mathrm{~N}$ & $\mathrm{~N}$ & $\mathrm{~N}$ & $\mathrm{~N}$ & $\mathrm{~N}$ & $\mathrm{~N}$ & $\mathbf{N}$ & $((?))$ \\
\hline 1-Chloro- $n$-octane & $\mathbf{N}$ & $\mathbf{S}$ & ( $\mathbf{S})$ & $(\mathbf{S})$ & $((\mathbf{S}))$ & $\mathrm{N}$ & $\mathrm{N}$ & $\mathrm{N}$ & $\mathrm{N}$ & $((?))$ & $(?)$ \\
\hline 1-Chloro- $n$-hexane & 80 & $\mathrm{~S}$ & $(\mathbf{S})$ & $(\mathrm{S})$ & $((\mathbf{S}))$ & $((?))$ & $(0)$ & (0) & $(0)$ & $\mathbf{S}$ & $\mathbf{S}$ \\
\hline Cyclohexanol & $\Theta=79$ & $\Theta=81$ & $\leftarrow$ & $\Theta=82$ & $\rightarrow$ & $\Theta=81$ & $\leftarrow$ & $\Theta=81$ & $\rightarrow$ & $\Theta=84$ & $\Theta=84$ \\
\hline 2-Methyl cyclohexanol & 28 & 55 & 35 & - & - & 47 & - & 35 & 34 & 54 & 57 \\
\hline 3-Methyl cyclohexanol & 71 & 91 & 75 & - & - & 84 & - & 75 & 76 & 90 & 93 \\
\hline 4-Methyl cyclohexanol & 66 & $\mathrm{~N}$ & 75 & - & - & (75) & - & 75 & (74) & 76 & $\mathrm{~N}$ \\
\hline Acetone & $\mathbf{S}$ & $\mathrm{N}^{\mathrm{b}}$ & $(\mathbf{S})$ & $(\mathbf{S})$ & $((\mathbf{S}))$ & $((\mathbf{S}))$ & $(\mathbf{S})$ & $(\mathrm{S})$ & $((\mathbf{S}))$ & $((\mathbf{S}))$ & $((\mathbf{S}))$ \\
\hline 2-Ethoxy ethanol & $\Theta=39$ & $\mathrm{~N}$ & $(\mathbf{S})$ & $(\mathrm{S})$ & $((\mathbf{S}))$ & $\Theta=70$ & $\leftarrow$ & $\Theta=81$ & $\rightarrow$ & $\mathrm{N}$ & $\mathrm{N}$ \\
\hline Acetonitrile & $\Theta=35$ & $\mathbf{N}$ & $((\mathbf{S}))$ & $((\mathbf{S}))$ & $((\mathbf{S}))$ & $((40))$ & $((30))$ & $((35))$ & $\mathbf{N}$ & $\mathbf{N}$ & $\mathrm{N}$ \\
\hline
\end{tabular}

a $\mathrm{N}$ denotes "not soluble" at $100^{\circ} \mathrm{C}$; $\mathrm{S}$ "soluble" at $-15^{\circ} \mathrm{C}$. Values in parentheses are ambiguous because of the gradual change in turbidity over a broad temperature range. ( ) shows the solution as being slightly turbid and $(())$ heavily turbid but with no phase separation above the temperature as indicated.

b Low-molecular-weight PST samples are soluble.

turbid solutions.

From the results shown in Table I, the diference between diblock and triblock copolymers is evident. Broadly speaking, PST-PMMA diblock samples are usually soluble in either type of selective solvents, i.e., those that dissolve either one of the parent homopolymers. On the other hand, PMMA-PST-PMMA triblock copolymers appear to be soluble in good solvents of PMMA but hardly soluble in nonsolvents of PMMA. Their solubility is more affected by the side PMMA-subchains than by the central PST-subchains. The detailed results are, of course, affected by various other factors such as molecular weight and composition of the samples.

\section{Intrinsic Viscosity Data}

The authors reported earlier an anomaly of [ $\eta$ ] data for PMMA-PST-PMMA triblock copolymers with nearly equimolar composition measured in $p$-xylene $(p \mathrm{XY})$ and 1-chloro- $n$ butane (1-CB). ${ }^{4,5}$ Both of them are $\Theta$-solvents toward PMMA but good solvents toward PST. In addition, PST and PMMA are only very poorly compatible in these solvents. ${ }^{4}$ For the sake of comparison, the behavior of PSTPMMA diblock copolymers in $p \mathrm{XY}$ at $30^{\circ} \mathrm{C}$ was examined. The results are shown in Table II. As noted before, ${ }^{4,5}$ the $[\eta]$ data of the two triblock samples with high molecular weights, 22B and 27B, were smaller than those of their precursor PST samples, $22 \mathrm{H}$ and $27 \mathrm{H}$, respectively.

No anomaly of this nature was observed for the diblock samples: their $[\eta]$ data were in most cases larger than those of their precursor PST-H. Instead they showed an anomaly of a different nature such that their solutions became visibly turbid and the values of the Huggins constant $k^{\prime}$ became very large (say, about 0.8 to 1.0 ). This anomaly appears to have resulted from either the intermolecular association or the micelle formation.

A comparison of a diblock and a triblock samples was also made in mixed solvent systems. For this purpose toluene (TOL), furfuryl alcohol $(\mathrm{FAL}),{ }^{8}$ and $p$-cymene ( $p \mathrm{CY}$ ) were selected; TOL is a good solvent for both PST and PMMA; FAL is a nonsolvent toward PST but a good solvent for PMMA; and $p C Y$ is the opposite. 
Table II. Intrinsic viscosities $[\eta]$ and Huggins constant $k^{\prime}$ of nearly equimolar PMMA-PST-PMMA triblock and PST-PMMA diblock copolymers compared with those of their precursor PST-H in $p$-xylene at $30^{\circ} \mathrm{C}$

\begin{tabular}{|c|c|c|c|c|c|c|c|c|}
\hline \multirow{2}{*}{ Code } & \multicolumn{3}{|c|}{ Precursor PST } & \multirow{2}{*}{ Code } & \multicolumn{4}{|c|}{ ST-MMA block copolymer } \\
\hline & $10^{-4} M_{w}$ & {$[\eta], \mathrm{d} l / \mathrm{g}$} & $k^{\prime}$ & & $10^{-4} M_{w}$ & $x^{\mathrm{a}}$ & {$[\eta], \mathrm{d} l / \mathrm{g}$} & $k^{\prime}$ \\
\hline & & & & & \multicolumn{4}{|c|}{ PMMA-PST-PMMA triblock type } \\
\hline $27 \mathrm{H}$ & 67.1 & 1.62 & 0.345 & 27B & 147.4 & 0.41 & 1.590 & 0.466 \\
\hline $22 \mathrm{H}$ & 38.0 & 1.09 & 0.343 & $22 \mathrm{~B}$ & 80.7 & 0.45 & 1.033 & 0.399 \\
\hline $26 \mathrm{H}$ & 13.6 & 0.556 & 0.329 & 26B & 30.8 & 0.46 & 0.582 & 0.402 \\
\hline \multirow[t]{2}{*}{$28 \mathrm{H}$} & 5.2 & 0.273 & 0.311 & 28B & 9.19 & 0.47 & 0.289 & 0.434 \\
\hline & & & & & \multicolumn{4}{|c|}{ PST-PMMA diblock type } \\
\hline $46 \mathrm{H}$ & 14.0 & 0.570 & 0.345 & $46 \mathrm{~B}$ & 30.0 & 0.45 & 0.660 & 0.92 \\
\hline $47 \mathrm{H}$ & 5.0 & 0.249 & 0.295 & 47B & 11.0 & 0.42 & 0.295 & 0.98 \\
\hline $52 \mathrm{H}$ & 3.3 & 0.195 & 0.338 & $52 \mathrm{~B}$ & 7.7 & 0.42 & 0.226 & 0.53 \\
\hline $39 \mathrm{H}$ & 2.7 & 0.176 & 0.270 & 39B & 5.5 & 0.48 & 0.192 & 0.52 \\
\hline
\end{tabular}

a ST-content by weight fraction.

Therefore, the TOL : FAL mixture gave a selective solvent system unfavorable toward PST; the TOL : $p C Y$ mixture gave a system unfavorable toward PMMA; and the $p C Y:$ FAL mixture a system having differing selectivities depending on its composition. The block copolymer samples examined were PST-PMMA 46B and PMMA-PST-PMMA 26B, both having nearly equimolar composition and nearly equal molecular weight ( $c f$., Table II). The reference homo-PST samples were PST-46H and PST-26H, which also have nearly equal molecular weights, $14 \times 10^{4}$ and $13.6 \times 10^{4}$, respectively. The homoPMMA samples were PMMA-31M and PMMA$38 \mathrm{M}$, whose molecular weights $M_{w}$ are $20.0 \times 10^{4}$ and $40.0 \times 10^{4}$, respectively. ${ }^{4}$

The relations of $[\eta]$ vs. solvent composition (by volume \%) are summarized in Figures $1-3$. The values of the Huggins constant $k^{\prime}$ of the block copolymer solutions are also shown. In the figure, filled circles identify the systems which gave rise to visibly turbid solutions. As judged from the changes in the solution turbidity and in the [ $\eta]$ and $k^{\prime}$ values with the solvent composition, the sample PST-PMMA $46 \mathrm{~B}$ appeared to undergo micelle formation in all the three systems beyond the solvent composition at which either one of the parent homopolymers became insoluble.

However, the behavior of the PMMA-PSTPMMA 26B was significantly different in the three mixed-solvent systems, depending on their

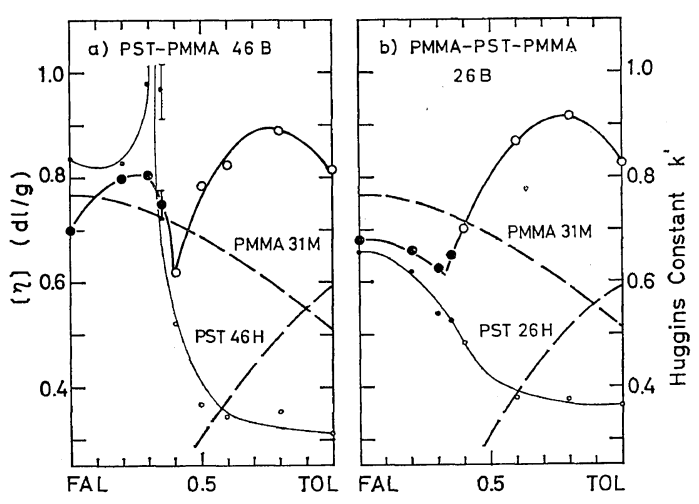

Figure 1. Viscometric behavior of (a) PSTPMMA diblock sample 46B and (b) PMMA-PSTPMMA triblock sample 26B in toluene (TOL) and furfuryl alcohol (FAL) mixture at $30^{\circ} \mathrm{C}$. Large circles denote [ $\eta]$ data and small circles $k^{\prime}$ data of the block copolymer solutions. Black circles identify the solutions which are visibly turbid. Heavy dashed curves show $[\eta]$ data of parent homopolymers as indicated; the data of PMMA $31 \mathrm{M}$ are shifted upward by $0.1(\mathrm{~d} l / \mathrm{g})$ unit for easier recognition.

degree of solvency. In the TOL : FAL mixture, its behavior was more or less similar to the PST-PMMA 46B. In the FAL-rich region it gave rise to visibly turbid solutions. However, their $[\eta]$ data were smaller than those of the PST-PMMA 46B, presumably reflecting the difference in their micelle sizes. In the TOL: $p C Y$ mixture, the value of $[\eta]$ gradually de- 

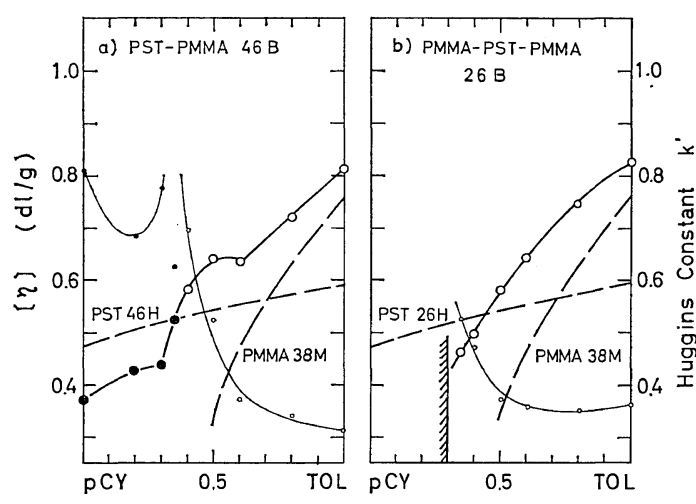

Figure 2. Viscometric behavior of (a) PSTPMMA diblock sample 46B and (b) PMMA-PSTPMMA triblock sample 26B in toluene (TOL) and $p$-cymene $(p \mathrm{CY})$ mixture at $30^{\circ} \mathrm{C}$. In the shaded region the block copolymer is insoluble. For other symbols, see Figure 1.

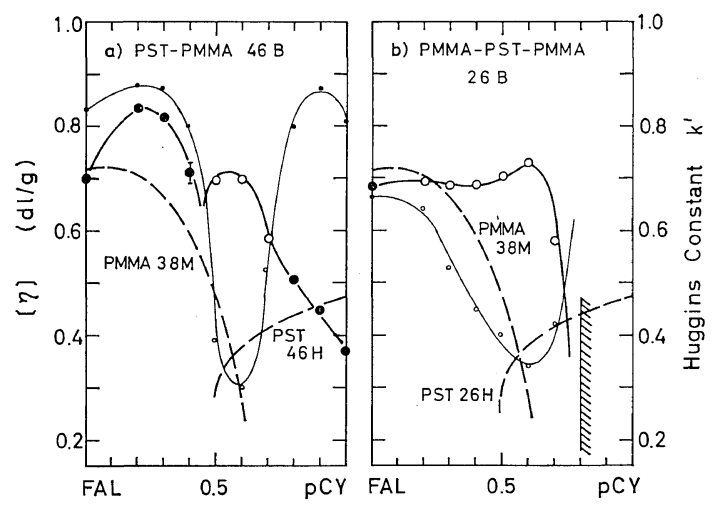

Figure 3. Viscometric behavior of (a) PSTPMMA diblock sample 46B and (b) PMMA-PSTPMMA triblock sample 26B in $p$-cymene $(p C Y)$ and furfuryl alcohol (FAL) mixture at $30^{\circ} \mathrm{C}$. For the symbols, see Figure 1 and 2.

creased with increasing $p \mathrm{CY}$-content and, at the $p \mathrm{CY}$-content of about $55 \%$, it became smaller than that of the precursor PST-26H but still gave a clear transparent solution. Finally the solution became turbid and the phase separation began to take place at a $p C Y$-content slightly above $65 \%$. In the $p C Y:$ FAL mixture, the PMMA-PST-PMMA 26B appeared to be soluble in the FAL-rich region, presumably forming stable micelles; but it became insoluble in the $p C Y$-rich region.

\section{Sedimentation Equilibrium Data}

The implication of the above results is that on certain occasions both of the PST-PMMA and PMMA-PST-PMMA block copolymers underwent micelle formation (intermolecular association). In order to examine how many block copolymer molecules are actually involved in such a micelle, an attempt was made to determine the apparent molecular weight $\bar{M}_{1}$ of the micelles by a sedimentation equilibrium method. ${ }^{16-21}$

First the two samples, PST-PMMA 46B and PMMA-PST-PMMA 26B, were examined in the TOL : $p C Y$ mixture by varying solvent composition. Application of the sedimentation equilibrium method to a binary solvent system requires a few additional consideration: ${ }^{20}$ one is concerned with the effect of solvent redistribution at the equilibrium, and the other with the effect of thermodynamic interaction between the polymer and one of the solvent i.e., in other words the effect of so-called preferential solvation. The latter is important also in the light-scattering measurement in binary-solvent systems. ${ }^{26,27}$ For convenience the two solvents are denoted as the principal solvent (componet 0 ) and the second solvent (component 2). However since the two solvents have nearly equal density (the density $\rho_{25}$ at $25^{\circ} \mathrm{C}$ is 0.8623 for TOL and 0.8533 for $p \mathrm{CY}$ ), virtually no redistribution of the solvents will take place: the effect of the solvent redistribution must be negligible. Therefore the expression of cellaveraged molecular weight, eq 1 , which was originally given for a single-solvent system, should be applicable to this binary-solvent system, by assuming the polymer to be centrifuged in a single solvent of the uniform density $\rho_{\mathrm{s}}$, which is given by

$$
\rho_{\mathrm{s}}=\rho_{0}+\left(1-\bar{v}_{2} \rho_{0}\right) c_{2}{ }^{0}
$$

Here $c_{2}{ }^{0}$ is the concentration of the second solvent 2 in the original (uniform) solution; $\rho_{0}$ is the density of the principal solvent 0 ; and $\bar{v}_{2}$ is the partial specific volume of the solvent $2 .^{20}$

In general, for the sedimentation equilibrium of a binary solvent system in which no redistribution of the solvents takes place, the cellaveraged molecular weight $\bar{M}_{1}^{\text {app }}$ (as calculated by eq 1) will give $\bar{M}_{1}^{*}$ in the limit of infinite 
dilution, ${ }^{20}$ which is defined as

$$
\lim _{c_{1} \rightarrow 0} \bar{M}_{1}^{\mathrm{app}}=\bar{M}_{1}^{*}=\bar{M}_{1}+\Gamma M_{2}\left(1-\bar{v}_{2} \rho_{\mathrm{s}}\right) /\left(1-\bar{v}_{1} \rho_{\mathrm{s}}\right)
$$

Here $M_{2}$ is the molecular weight of the second solvent $2 ; \Gamma$ is the so-called solvation parameter related to the amount of moles of the solvent 2 preferentially solvated to one mole of the polymer 1 ; and $\bar{v}_{1}$ is the partial specific volume of the polymer 1 (therefore equivalent to $\bar{v}^{0}$ in eq 2). In principle, $\bar{M}_{1}$ cannot be determined, unless $\Gamma$ is known. However, again because of the solvent densities of this particular mixture being nearly equal, the term $\left(1-\bar{v}_{2} \rho_{\mathrm{s}}\right)$ becomes virtually zero: consequently the quantity $\bar{M}_{1}^{*}$ is practically indistinguishable from the quantity $\bar{M}_{1}$. If micelles formed in a block copolymer-binary solvent mixture are stable even in the limit of zero polymer concentration, the quantity $\bar{M}_{1}^{*}$ becomes nearly equal to $\bar{M}_{1}$, which in turn reflects the molar weight of such micelles.

The results of the TOL: $p C Y$ systems are summarized in Figiure 4, where values of $M_{w} / \bar{M}_{1}^{\text {app }}$ are plotted against original polymer

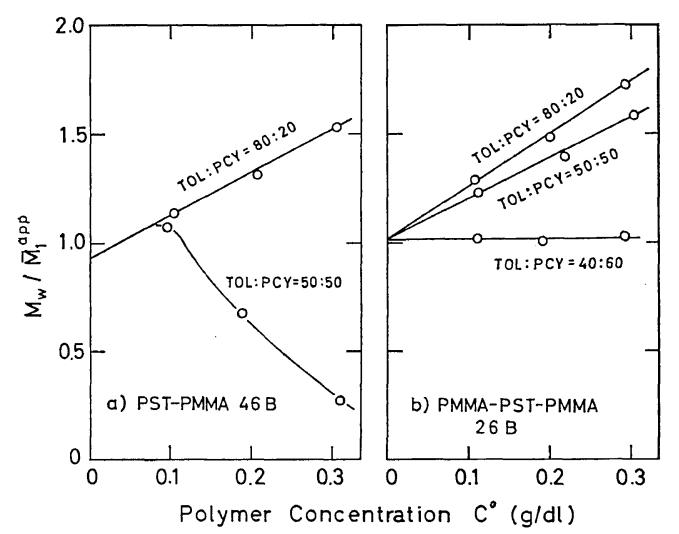

Figure 4. Sedimentation equilibrium data as shown by the ratio $M_{w} / \bar{M}_{1}^{\text {app }}$ versus original polymer concentration $c^{0}$ of (a) PST-PMMA diblock sample 46B and (b) PMMA-PST-PMMA triblock sample 26B in toluene (TOL) and p-cymene $(p C Y)$ mixture with various volume ratios as indicated. Centrifugation speed $\omega$ was $6,020 \mathrm{rpm}$ $(\lambda \cong 0.6-0.7)$ except for one case of PST-PMMA $46 \mathrm{~B}$ in 50/50-mixture, for which $\omega=2,547(\lambda \cong$ $0.11-0.12$ ). All the measurements were made at $30^{\circ} \mathrm{C}$. concentration $c^{0}$. From Figure $4 \mathrm{~b}$, it is seen that the value $\bar{M}_{1}^{*}$ of the triblock sample $26 \mathrm{~B}$ is nearly equal to $M_{w}$ even in a mixture of the $p$ CY-content as much as 60\%: this suggests that no micelle formation was taking place up to this $p C Y$-content. It should be remembered that the $[\eta]$ anomaly in this mixture $(c f$., Figure $2 \mathrm{~b})$ had already been found. When the $p C Y$ content was increased to $65 \%$, the value $\bar{M}_{1}{ }^{*}$ became unmeasurably large: even a centrifugation with a rotor speed as low as $2,007 \mathrm{rpm}$ forced all the polymer molecules to sediment rapidly down to the cell bottom. When the $p$ CY-content was increased slightly over $65 \%$, a macroscopic phase separation took place.

From Figure $4 \mathrm{a}$, it can be seen that the value $\bar{M}_{1}{ }^{*}$ of the diblock sample $46 \mathrm{~B}$ is nearly equal to its true $M_{w}$, when the measurement is made in a mixture of $20 \% p \mathrm{CY}$-content. However when the $p C Y$-content is $50 \%$, the $M_{w} / \bar{M}_{1}^{\text {app }}$ vs. $c^{0}$ plot shows a feature characteristic of a self-associating system, ${ }^{28}$ i.e., the value of $\bar{M}_{1}^{\text {app }}$ rapidly increases with increasing $c^{0}$, this being indicative of reversible association-dissociation of solute polymers. In this mixture the $[\eta]$ value takes a maximum (cf., Figure 2 a). As the $p C Y$-content is increased to $60 \%$, the value of $\bar{M}_{1}^{*}$ of the diblock sample $46 \mathrm{~B}$ tends to be extremely large, as opposed to the triblock sample 26B. Its sedimentation behavior suggests that the molar weight of micelles should be larger than $10^{7}$, although unfortunately the exact value could not be determined. From similar measurements in the TOL : FAL mixture of $65 \%$ FAL-content, which gave rise to visibly turbid solutions for both of the samples 26B and 46B ( $c f$., Figures $1 \mathrm{a}$ and $\mathrm{b}$ ), their cellaveraged molecular weights were found to be unmeasurably large, suggesting the formation of very large size micelles. Again unfortunately the difference in their micelle sizes could not be distinguished.

The sedimentation experiments were carried out also in a single solvent system, $p \mathrm{XY}$ at $30^{\circ} \mathrm{C}$. The results are listed in Table III. The values of $\bar{M}_{1}$ of all the triblock samples were found to be nearly equal to their true $M_{w}$ values: this implies that all the triblock samples did not undergo micelle formation in $p \mathrm{XY}$ at $30^{\circ} \mathrm{C}$. On the contrary, the results of the 
Table III. Sedimentation equilibrium and lightscattering apparent-molecular weight data of nearly equimolar PST-PMMA diblock and PMMA-PST-PMMA triblock copolymers in $p$-xylene at $30^{\circ} \mathrm{C}^{\text {a }}$

\begin{tabular}{lrlllr}
\hline Code & $10^{-4} M_{w}$ & $\bar{v}^{0}$ & $10^{-4} \bar{M}_{1}^{\mathrm{b}}$ & $10^{5} \lambda^{\mathrm{c}}$ & $10^{-4} M_{\mathrm{app}}^{\mathrm{d}}$ \\
\hline PST-PMMA & diblock copolymers & \\
46B & 30.0 & 0.8585 & 250 & $(0.11 \sim 0.17)$ & 266.5 \\
47B & 11.0 & 0.8556 & 15 & $(1.2 \sim 1.5)$ & 62.0 \\
39B & 5.5 & 0.8623 & 5.4 & $(2.57 \sim 3.54)$ & 4.9 \\
PMMA-PST-PMMA & triblock copolymers \\
27B & 147.4 & 0.8545 & - & - & 149.5 \\
22B & 80.7 & 0.8585 & - & - & 78.3 \\
26B & 30.8 & 0.8585 & 24.5 & $(0.58 \sim 0.73)$ & 31.1 \\
\hline
\end{tabular}

a The value of $\rho_{\mathrm{S}}=0.8523$ was used for the solvent density of $p \mathrm{XY}$ at $30^{\circ} \mathrm{C}$.

b The quantity $\bar{M}_{1}$ denotes cell-averaged molecular weight (eq 1) extrapolated to zero polymer concentration; the extrapolation with respect to $\lambda^{2}$ was not performed, and this may have caused the ambiguity in determining $\bar{M}_{1}$.

c The range of $\lambda$-values evaluated for each centrifugation condition.

d The quantity $M_{\text {app }}$ denotes light-scattering apparent molecular weight extrapolated to zero polymer concentration: the $M_{\mathrm{app}}$ should correspond to $\bar{M}_{1}$ in the limit of zero centrifugation speed, i.e., $\lambda^{2} \rightarrow 0$.

diblock samples were different from one another, depending on their molecular weights.

For the sample 46B which had the largest molecular weight among those examined here, the value of $\bar{M}_{1}$ (estimated by extrapolating values of $\bar{M}_{1}^{\text {app }}$ obtained in a range of different $\lambda$ to zero concentration) did not seriously differ from that of $M_{\text {app }}$ (determined by light scattering in the same solvent) as seen in Table III. However the value of $\bar{M}_{1}$ was found to be about 7 to 10 times larger than its true $M_{w}$. This result implies formation of stable micelles, each consisting of 7 to 10 block copolymer molecules. By the term "stable" it is meant that the micelle size is practically unchanged almost independent of the rotor speed $\omega$, thus of $\lambda$, and of the original concentration $c^{0}$, so far as the concentration range examined is concerned. For the sample 47B having the second largest $M_{w}$, the behavior is more complicated. The value of $\bar{M}_{1}$ (estimated in the same way as for
46B) was significantly smaller than that of $M_{\mathrm{app}}$ (obtained by light scattering) but rather near to its true $M_{w}$. However the sedimentation behavior of this sample in $p \mathrm{XY}$ was observed to be strongly dependent on the rotor speed $\omega$, as mentioned below. For example, high-speed centrifugation gave rise to fringe patterns which were indicative of the accumulation of large amounts of solute macromolecules near the cell bottom; whereas lowering the speed gave rise to the patterns with slight fringe displacement, this indicating that the accumulated solute molecules had been redispersed throughout the whole solution column as a consequence of micelle dissociation. The change in $c^{0}$ also gave similar sedimentation patterns. All these characteristics apper to be similar to those of a self-associating system. ${ }^{28}$ The light scattering apparent molecular weight, which may roughly be equivalent to $\bar{M}_{1}$ determined in the limit of zero centrifugation speed, was found for this sample $47 \mathrm{~B}$ to be about 6 times larger than its true $M_{w}$. These results suggest the formation of unstable or reversibly associating-dissociating micelles, which are different from those for 46B.

On the other hand, for the other samples, $39 \mathrm{~B}$ and 52B*, with lower molecular weights, no anomalies were observed: The sedimentation behavior of these samples were normal and independent of $\omega$ and $c^{0}$, and the value of $\bar{M}_{1}$ obtained for $39 \mathrm{~B}$ was found to be nearly equal to its true $M_{w}$. The results imply that there was no micelle formation taking place. In short, in $p \mathrm{XY}$ at $30^{\circ} \mathrm{C}$ the low-molecular-weight diblock samples did not form micelles, whilst the samples having large $M_{w}$ (say, above $10^{5}$ ) could form micelles, whose stability increased as the $M_{w}$ increased. A similar test was also performed in diethyl malonate which has opposite solvency to $p \mathrm{XY}$. In this case, however, measurements of $\bar{M}_{1}^{\text {app }}$ were difficult, because the difference in density between the block copolymers and the solvent diethyl malonate is not very large. Therefore light-scattering apparent molecular weights were determined. The measurements were only made on the two samples, PST-PMMA 46B and PMMA-PST-

\footnotetext{
* Data of $\bar{M}_{1}^{\text {app }}$ for this sample were strongly scattered so that no exact value of $\bar{M}_{1}$ could be evaluated.
} 
PMMA 26B. The values determined at $30^{\circ} \mathrm{C}$ were found to be nearly equal to their true $M_{w}$, implying no micelle formation. However upon lowering the temperature to, say, $15^{\circ} \mathrm{C}$, the macroscopic phase separation took place in solutions of the both samples.

\section{DISCUSSION}

All the above results indicate that the diblock and triblock copolymers behave quite differently especially in certain selective solvents. In general the PST-PMMA diblock samples appear to form micelles in either types of selective solvents: presumably the insoluble subchains accumulate in the core of each micelle, whilst the soluble subchains wrap around the core and stabilize the whole micelle by preventing from further aggregation. Evidence from other sources such as the adsorption behavior of ST-MMA block copolymers on activated silicagel surfaces ${ }^{29,30}$ supports this conclusion on the micelle structure. The size and the stability of such micelles are, of course, critically dependent on the molecular weight and composition of the block copolymers as well as on the nature of the solvents.

On the contrary the behavior of the PMMAPST-PMMA triblock copolymers appears to be more critically influenced by the nature of the solvents. In the selective solvents having unfavorable solvency toward central PST-subchains, their behavior is more or less similar to the diblock copolymers. On the other hand, in the selective solvents having unfavorable solvency toward side PMMA-subchains, the triblock copolymers are less stable and they are usually more likely to undergo macroscopic phase separation ( $c f$., Table I). Occasionally they form gel-like structures with increasing polymer concentration. $^{31}$ In the region in between the stable solution and the macroscopic phase separation states, which might be achieved by changing solvent composition (e.g., for TOL: $p C Y$ binary mixture) and by changing temparature (e.g., for $p \mathrm{XY}$ system), the triblock copolymers often exhibit anomalous $[\eta]$ behavior such as those described above. The evidence from sedimentation equilibrium and light-scattering studies suggests that, under such conditions, the triblock copolymers do not undergo intermolecular association but remain in the state of monomolecular dispersion ( $c f$., Table III). The $[\eta]$ anomalies must therefore be attributed to the conformational anomaly of the individual triblock copolymer chains. In previous articles ${ }^{4,5}$ the possibility of intramolecular association of two side PMMA-subchains within a single triblock chain has been suggested. The implication was as follows: in view of the fact that two of the PST-PMMA diblock samples, 46B and $47 \mathrm{~B}$, could form micelles in $p \mathrm{XY}$, each of the two side PMMA-subchains of, at least, three triblock samples, $(27 \mathrm{~B}, 22 \mathrm{~B}$, and 26B), have molecular weights large enough to undergo micelle formation in the same solvent. Nevertheless, the apparent molecular weight data show that all the triblock copolymers do not undergo intermolecular micelle formation. Therefore the possibility remains that two PMMA-subchains within an individual triblock copolymer chain undergo micelle formation, whilst the central PST-subchain wraps around the collapsed PMMA-subchains. This feature would explain the $[\eta]$ anomaly as well as the sedimentation equilibrium behavior. However this suggestion demands a further elaboration on a few additional aspects.

First the well-known Flory-Fox equation ${ }^{25}$ is also assumed to be valid for block copolymer solutions.

$$
[\eta]=\Phi\left\langle r^{2}\right\rangle^{3 / 2} / M
$$

Here $\Phi$ is the Flory-Fox viscosity constant ${ }^{25}$ and $\left\langle r^{2}\right\rangle$ is the mean-square end-to-end distance. In addition, if the collapsed PMMA-subchains are assumed to contribute nothing to the overall dimension of the block copolymer chain (while the PST-subchain remains expanded having the dimension the same as that of the precursor PST-H in the same solvent) the value of $\left\langle r^{2}\right\rangle$ in eq 6 may be replaced by the value $\left\langle r_{\mathrm{A}}{ }^{2}\right\rangle$ of the precursor PST-H. Assuming that eq 6 is still valid for such block-copolymer chains with anomalous conformations, [i] of the block copolymer can be correlated with $[\eta]_{\mathrm{A}}$ of the precursor PST-H as

$$
[\eta] /[\eta]_{\mathrm{A}}=M_{\mathrm{A}} / M=x .
$$

This equation implies that for a nearly equimolar 
block copolymer of PMMA-PST-PMMA type the value of $[\eta]$ could become as small as onehalf of $[\eta]_{A}$, without introducing the assumption of intramolecular association of two PMMAsubchains: the reduction of $[\eta]$ of the triblock copolymer is a consequence of an obvious situation where the collapsed PMMA-subchains might not contribute to the overall dimension but must do to the overall mass of the block copolymer chain. Then, this equation, eq 7, should hold for PST-PMMA diblock copolymers, provided they do not form micelles.

The situation as mentioned above represents, of course, a hypothetical limiting case which is rather unlikely to exist. A slightly more realistic calculation of the contribution of, say, B-subchain(s) exposed to their nonsolvent to the overall dimensions of AB-diblock or BABtriblock copolymers may be carried out ${ }^{32-35}$ by applying the perturbation method. ${ }^{36}$ The results of such calculations showed that the value of $[\eta]$ of a BAB-triblock copolymer might become comparable to, or even smaller than, $[\eta]_{\mathrm{A}}$ of its precursor homopolymer A under certain limited conditions, which are rather unlikely but still conceivable, without introducing the assumption of intramolecular association of the two B-subchains. ${ }^{34}$ In other words, the conditions are such wherein the contribution of the collapsed B-subchains to the overall dimension of the whole block copolymer chain becomes trivial in relation to that of the A-subchain.

In this connection it is interesting to note the sedimentation-velocity behavior of the BABtriblock copolymers in $p \mathrm{XY}$ solutions. As is well known, the sedimentation coefficient $s$ of a polymer with $M$ and the partial specific volume $\bar{v}$ can be related to the (translational) friction coefficient $f$ by the celebrated Svedberg equation: ${ }^{37}$

$$
s=M(1-\bar{v} \rho) / N f
$$

where $N$ is the Avogadro number; and $\rho$ is the solution density and is identified with the solvent density $\rho_{\mathrm{S}}$ in the limit of zero polymer concentration. Based on the same assumptions employed for the $[\eta]$ equation (i.e., eq 6), Flory $^{25}$ suggested a relation

$$
f=\eta_{\mathrm{s}} \boldsymbol{P}\left\langle\boldsymbol{r}^{2}\right\rangle^{1 / 2}
$$

where $\eta_{\mathrm{s}}$ is the solvent viscosity and $\boldsymbol{P}$ is the Flory constant. Again eq 9 is assumed to be valid for block copolymer solutions and the same approximation is introduced as to eq 7, i.e., that the collapsed B-subchains contribute nothing to the overall dimension of the block copolymer chain and, hence, the value of $\left\langle r^{2}\right\rangle$ in eq 9 can be replaced by $\left\langle r_{\mathrm{A}}{ }^{2}\right\rangle$. Then the same value of $f$ for both the block copolymer and the precursor homopolymer $\mathrm{A}$ is obtained. On the other hand, if it is arbitrarily assumed that the value of $\left\langle r_{A}{ }^{2}\right\rangle$ is half of the value of $\left\langle r^{2}\right\rangle$, then the ratio of $f$ would be $f / f_{\mathrm{A}}=1.414$. Sedimentation velocity experiments ${ }^{38}$ showed that the BAB-triblock sample 27B and its precursor PST-27H have $s=16.69$ and 9.89 (in Svedberg unit), respectively, in $p \mathrm{XY}$ at $30^{\circ} \mathrm{C}$. From these values of $s$ and the buoyancy factor $\left(1-\bar{v} \rho_{\mathrm{s}}\right)$ of these systems $(c f$. , Table III), the ratio of $f$ was found to be $f / f_{A}=1.45$. The results imply that the PMMA-subchains contribute to the overall dimension of the block copolymer chain to a considerable extent.

All the above arguments on the values of $[\eta]$ and $f$ of a BAB-triblock copolymer in relation with those of its precursor homopolymer A should be applicable to an AB-biblock copolymer as well, provided that the latter does not undergo the intermolecular association. Thus the $[\eta]$ of an AB-diblock copolymer could be smaller than the $[\eta]_{\mathrm{A}}$ of its precursor homopolymer A, without forming micelles. However, no such examples were found in $p \mathrm{XY}$ solutions of the nearly equimolar PST-PMMA diblock copolymers examined here. Instead, they often formed micelles. The question arising is then why the block copolymers should undergo the micelle formation, whilst the triblock copolymers having PMMA-subchains of comparable or even larger sizes do not undergo the intermolecular association.

In view of these arguments, the experimental data available to date appear to disfavour (but do not completely rule out) a simplified picture of the conformation of a BAB-triblock copolymer chain which the collapsed B-subchains would not undergo the intramolecular association but simply make a trivial contribution to its overall dimension. However, one should hasten to add that the hydrodynamic data alone cannot 
provide decisive evidence as to whether or not the intramolecular association of the two Bsubchains within a single block-copolymer chain of BAB-type is really taking place.

Acknowledgement. The authors wish to thank Dr. Hiroshi Ohnuma for his contribution to the early stage of this study, and Mr. Nobuo Donkai for his assistance in sedimentation equilibrium experiments. They also wish to thank Dr. Teruo Fujimoto of Nagoya University for his valuable help in the art of anionic polymerization. A part of this work was supported by the Ministry of Education under Grant \#A 4037.

\section{REFERENCES}

1. T. Kotaka, H. Ohnuma, and Y. Murakami, J. Phys. Chem., 70, 4099 (1966).

2. H. Ohnuma, T. Kotaka, and H. Inagaki, Polymer, 10, 501 (1969).

3. T. Kotaka, H. Ohnuma, and H. Inagaki, ibid., 10, 517 (1969).

4. H. Ohnuma, T. Kotaka, and H. Inagaki, Polymer J., 1, 716 (1970).

5. T. Kotaka, H. Ohnuma, and H. Inagaki, Polymer Preprints (Div. Polym. Chem., Amer. Chem. Soc.), 11, 660 (1970).

6. T. Kotaka, T. Tanaka, H. Ohnuma, Y. Murakami, and H. Inagaki, Polymer J., 1, 245 (1970).

7. See for example (a) Y. Gallot, M. Leng, H. Benoit, and P. Rempp, J. Chim. Phys., 59, 1093 (1962); (b) S. Ye. Bresler, L. M. Pyrkov, S. Yu. Frenkel, L. A. Laius, and S. I. Klenin, Vysokomol. Soedin., 4, 250 (1962); (c) G. E. Molau, J. Polym. Sci., Part-A, 3, 1267 (1965); ibid., Part- $A$, 3, 4235 (1965).

8. M. Fukuda, Thesis for the Degree of Master of Engineering, Kyoto Univ., 1968; M. Fukuda, H. Utiyama, and M. Kurata, private communication.

9. M. Szwarc, Fortschr. Hochpolym. Forsch., 2, 275 (1960).

10. M. Baer, J. Polym. Sci., Part-A, 2, 417 (1964).

11. J. R. Urwin and J.M. Stearne, Makromol. Chem., 78, 197 (1964).

12. D. Freyss, P. Rempp, and H. Benoit, J. Polym. Sci., Part-B, 2, 217 (1964).

13. T. Fujimoto, N. Ozaki, and M. Nagasawa, ibid., Part-A, 3, 2259 (1965).

14. H. Inagaki, H. Suzuki, M. Fujii, and T. Matsuo, J. Phys. Chem., 70, 1718 (1966).

15. T. Kotaka, Y. Murakami, and H. Inagaki, ibid., 72, 829 (1968).

16. K. E. Van Holde and R. L. Baldwin, J. Phys. Chem., 62, 734 (1958).

17. R. L. Baldwin and K. E. Van Holde, Fortschr. Hochpolym. Forsch., 1, 451 (1960).

18. F. E. LaBar and R. L. Baldwin, J. Phys. Chem., 66, 1952 (1962).

19. D. A. Yphantis, Biochemistry, 3, 297 (1964).

20. H. Fujita, "Mathematical Theory of Sedimentation Analysis," Academic Press, New York, N. Y., 1962.

21. T. Kotaka, N. Donkai, and H. Inagaki, $J$. Polym. Sci., Part A-2, 9, 1379 (1971).

22. T. Kotaka, N. Donkai, H. Ohnuma, and H. Inagaki, ibid., Part-A2, 6, 1803 (1968).

23. W. H. Stockmayer, L. D. Moore, Jr., M. Fixman, and B. N. Epstein, ibid., 16, 517 (1955).

24. M. Leng and H. Benoit, ibid., 57, 263 (1962).

25. P. J. Flory, "Principles of Polymer Chemistry," Cornell Univ. Press, Ithaca, N. Y., 1953, Chapters 12 and 13.

26. J. G. Kirkwood and R. J. Goldberg, J. Chem. Phys., 18, 54 (1950).

27. W. H. Stockmayer, ibid., 18, 58 (1950).

28. See for example: E. T. Adams, and H. Fujita, "Ultracentrifugal Analysis in Theory and Experiment," J.W. Williams Ed., Academic Press, New York, N. Y., 1963.

29. F. Kamiyama, H. Matsuda, and H. Inagaki, Makromol. Chem., 125, 268 (1969).

30. F. Kamiyama, H. Inagaki, and T. Kotaka, Polymer J. 3, No. 4 (1972), in press.

31. T. Tanaka and T. Kotaka, unpublished experiments.

32. M. Leng and H. Benoit, J. Chim. Phys., 59, 929 (1962).

33. D. Froelich and H. Benoit, Makromol. Chem., 92, 224 (1966).

34. T. Tanaka and T. Kotaka, Bull. Inst. Chem. Res., Kyoto Univ., in press.

35. H. Sato and K. Kamada, Bull. Chem. Soc., Japan, 40, 2264 (1967).

36. M. Fixman, J. Chem. Phys., 23, 1656 (1955).

37. Th Svedberg and K. O. Pedersen, "The Ultracentrifuge," Oxford University Press, London, 1940, Chapter B.

38. H. Ohnuma and T. Kotaka, unpublished experiments. 